\title{
FRACTIONAL INTEGRALS OF STATIONARY PROCESSES AND THE CENTRAL LIMIT THEOREM
}

\author{
M. ROSENBLATT, University of California, San Diego
}

\begin{abstract}
A class of limit theorems involving asymptotic normality is derived for stationary processes whose spectral density has a singular behavior near frequency zero. Generally these processes have 'long-range dependence' but are generated from strongly mixing processes by a fractional integral or derivative transformation. Some related remarks are made about random solutions of the Burgers equation.
\end{abstract}

FRACTIONAL. INTEGRAL; STATIONARY PROCESS: CENTRAL LIMIT THEOREM; SINGU. LAR SPECTRUM; BURGERS EQUATION

\section{Introduction}

Let $X(t),-\infty<t<\infty$, be a strictly stationary real-valued separable random process with $E X(t) \equiv 0$ and $E X(t)^{4}<\infty$. Our interest is in limit theorems for

$$
A(T)=\int X(t) g(t / T) d t
$$

as $T \rightarrow \infty$, where $g$ is a real-valued integrable function. The standard central limit theorem is for

$$
g(t)=\left\{\begin{array}{cc}
1 & \text { if } \quad 0<t<1, \\
0 & \text { otherwise }
\end{array}\right.
$$

and is often proved under a number of possible strong mixing conditions (see [4] and [5]). Suppose that the process $X(t)$ has an absolutely continuous spectral distribution function $F$ with spectral density $f$. The usual assumption on the variance of (1) for $g$ given by (2) is that it is proportional to $T$ as $T \rightarrow \infty$. In order to arrive at this rate of growth it is usual to assume that

$$
\lim _{\lambda \rightarrow 0} f(\lambda)=c>0
$$

However, there are a number of special results in which asymptotic normality has been obtained where the spectral density has a behavior like

Received 2 February 1976.

Research carried out while the author was visiting the Australian National University and supported in part by the Office of Naval Research. 


$$
f(\lambda) \simeq|\lambda|^{-\alpha}, \quad \alpha<1
$$

as $|\lambda| \rightarrow 0$ (see [1], [3] and [7]). If $\alpha$ is not an even integer, such processes are not strongly mixing (see [2]). Our object is to obtain a broader class of such limit theorems in which the basic behavior is laid out in a way that may be more apparent. The argument is based on the assumption that $X(t)$ can be identified as a fractional integral of a strongly mixing process and $g$ as a fractional derivative of a function.

We shall want to specify conditions on the process $X(t)$ in terms of an auxiliary stationary process $Y(t)$. The conditions on $Y(t)$ will be those used in the theorem of [5]. Let $\mathscr{B}_{t}(Y)$ be the Borel field generated by the random variables $Y(\tau), \tau \leqq t$, and $\mathscr{F}_{t}(Y)$ the Borel field generated by the random variables $Y(\tau), \tau \geqq t$. We shall say that the process $Y$ is strongly mixing if

$$
\sup _{\substack{B \in \mathfrak{s}_{i} \\ F \in \mathcal{F}_{r}}}|P(B \cap F)-P(B) P(F)|=g(\tau-t) \downarrow 0
$$

as $\tau-t \rightarrow \infty$. The process $Y$ will be said to satisfy Assumption A if the following conditions are fulfilled.

Assumption A.

(a) $Y$ is a stationary separable process with $E Y(t) \equiv 0, E Y(t)^{4}<\infty$, and $E|Y(t)-Y(\tau)|^{4} \rightarrow 0$ as $t-\tau \rightarrow 0$.

(b) $Y$ is strongly mixing.

(c) The covariance function $R(t)$ of $Y, R\left(t_{2}-t_{1}\right)=E\left[Y\left(t_{2}\right) Y\left(t_{1}\right)\right]$, is absolutely integrable with the spectral density $f(\lambda)$ of $Y$,

$$
f(\lambda)=\frac{1}{2 \pi} \int e^{-i \lambda t} R(t) d t
$$

positive everywhere.

(d) The fourth-order cumulant function of $Y$,

$$
\begin{aligned}
Q\left(t_{2}-t_{1}, t_{3}-t_{1}, t_{4}-t_{1}\right)= & E\left[Y\left(t_{1}\right) Y\left(t_{2}\right) Y\left(t_{3}\right) Y\left(t_{4}\right)\right] \\
& -R\left(t_{2}-t_{1}\right) R\left(t_{4}-t_{3}\right)-R\left(t_{3}-t_{1}\right) R\left(t_{4}-t_{2}\right) \\
& -R\left(t_{4}-t_{1}\right) R\left(t_{3}-t_{2}\right),
\end{aligned}
$$

is absolutely integrable.

Notice that under Assumption A the spectral density $f$ is continuous as is the fourth-order cumulant spectral density,

$$
q\left(\lambda_{1}, \lambda_{2}, \lambda_{3}\right)=\iiint \exp \left(-i\left\{t_{1} \lambda_{1}+t_{2} \lambda_{2}+t_{3} \lambda_{3}\right\}\right) Q\left(t_{1}, t_{2}, t_{3}\right) d t_{1} d t_{2} d t_{3} .
$$


The notion of a fractional integral (or derivative), when meaningful, will be of some interest. Because of the concern with stationarity and time translation, the Weyl definition of a fractional integral seems to be most appropriate (see [8], Vol. 2, p. 133). Let us call

$$
\begin{aligned}
\left(I_{c} Y\right)(t) & =\frac{1}{\Gamma(\alpha)} \int_{-\alpha}^{t}(t-\tau)^{\alpha \cdot 1} Y(\tau) d \tau \\
& =\operatorname{lim.m.~}_{L \rightarrow \infty} \frac{1}{\Gamma(\alpha)} \int_{L .}^{t}(t-\tau)^{\alpha-1} Y(\tau) d \tau
\end{aligned}
$$

the $\alpha$ th fractional integral, $0<\alpha<1$, of $Y$ if it is well-defined. Our definition is understood to be given by the limit in mean square on the right of Equation (5). One can define

$$
\left(I_{1} Y\right)(t)=\underset{\alpha \uparrow 1}{\operatorname{li.i.m}}\left(I_{\alpha} Y\right)(t) .
$$

An alternative equivalent definition is given by

$$
\left(I_{1} Y\right)(t)=\underset{L \rightarrow \text { i.m. }}{\lim } \frac{1}{L} \int_{0}^{L} \int_{-s}^{t} Y(\tau) d \tau d s
$$

Then, recursively, one can define $I_{\beta} Y$ for all $\beta>0$ for which it exists by

$$
I_{1+\beta} Y=I_{1}\left(I_{\beta} Y\right), \quad \beta>0 .
$$

The fractional derivative $D^{\alpha} Y, 0<\alpha<1$, is given by

$$
D^{\alpha} Y=D^{1}\left(I_{1} \text { a } Y\right)
$$

where by $D^{1}$ one understands the derivative in mean square. The derivative $D^{\alpha}$ for all $\alpha>0$ would then be given recursively when it exists by

$$
D^{1+\alpha} Y=D^{1}\left(D^{\alpha} Y\right), \quad \alpha>0 .
$$

It is then clear that one could just as well make the identification

$$
I_{\alpha}=D^{-\alpha}
$$

for all $\alpha$. These definitions could be extended slightly to that of fractional integrals (derivatives) centred at frequency $\mu$. Thus the $\alpha$ th fractional integral $I_{\alpha, \mu}$ centred at frequency $\mu$ would be given by

$$
\left(I_{\alpha, \mu} Y\right)(t)=\frac{1}{\Gamma(\alpha)} \int_{-x}^{t}(t-\tau)^{\alpha-1} e^{i \mu \tau} Y(\tau) d \tau
$$

for $0<\alpha<1$ with the obvious modification given for other values of $\alpha$ as in the case of an ordinary fractional integral (or derivative).

Lemma 1. Let $Y$ be a separable weakly stationary process $(E Y(t) \equiv 0)$ 


$$
\begin{gathered}
Y(t)=\int_{-\infty}^{\infty} e^{i t \lambda} d Z_{Y}(\lambda) \\
E d Z_{Y}(\lambda) d Z_{Y}(\mu)=\delta(\lambda+\mu) d F_{Y}(\lambda)
\end{gathered}
$$

with corresponding process $Z_{Y}$ of orthogonal increments and spectral distribution function $F_{Y}$. The ath $(0 \leqq \alpha)$ fractional integral (derivative) $I_{\alpha, \mu} Y\left(D^{\alpha, \mu} Y\right)$ centred at $\mu$ exists as a weakly stationary process if and only if

$$
\begin{aligned}
& \int|\lambda-\mu|^{-2 \alpha} d F_{Y}(\lambda)<\infty \\
& \left(\int|\lambda-\mu|^{2 \alpha} d F_{Y}(\lambda)<\infty\right)
\end{aligned}
$$

and is given by

$$
\begin{aligned}
& \left(I_{\alpha, \mu} Y\right)(t)=\int(i(\lambda-\mu))^{-\alpha} e^{i t(\lambda-\mu)} d Z_{Y}(\lambda) \\
& \left(\left(D^{\alpha, \mu} Y\right)(t)=\int(i(\lambda-\mu))^{\alpha} e^{i t(\lambda-\mu)} d Z_{Y}(\lambda)\right) .
\end{aligned}
$$

It will be enough to go through the argument for $I_{\alpha, \mu} Y$ with $0<\alpha<1$. Then

$$
\begin{aligned}
\left(I_{\alpha, \mu} Y\right)(t) & =\underset{L \rightarrow \infty}{\lim . \operatorname{lo}} \frac{1}{\Gamma(\alpha)} \int_{-L}^{t}(t-\tau)^{\alpha-1} e^{-i \mu \tau} Y(\tau) d \tau \\
& =\int_{-\infty}^{\infty} \frac{1}{\Gamma(\alpha)} \int_{-\infty}^{t} e^{i \tau(\lambda-\mu)}(t-\tau)^{\alpha-1} d \tau d Z_{Y}(\lambda)
\end{aligned}
$$

But

$$
\int_{-\infty}^{t} e^{i \tau(\lambda-\mu)}(t-\tau)^{\alpha-1} d \tau=\Gamma(\alpha) e^{i t(\lambda-\mu)}(i(\lambda-\mu))^{-\alpha}
$$

The following result is a useful formal analogue of integration by parts.

Lemma 2. Let $Y(t)$ be a separable weakly stationary process, $E Y(t) \equiv 0$, and $g(t)$ a bounded integrable function. Then if $\left(I_{\alpha} Y\right)(t)$ exists, $0<\alpha<1$, and if

$$
g_{T}(t)=g(t / T)
$$

it follows that

$$
\int\left(I_{\alpha} Y\right)(t) g_{T}(t) d t=\int Y(t)\left(\tilde{I}_{\alpha} g_{T}\right)(t) d t
$$

where

$$
\begin{aligned}
\left(\tilde{I}_{\alpha} g_{T}\right)(t) & =\int_{-t}^{\infty}(t+\tau)^{\alpha-1} g\left(\frac{\tau}{T}\right) d \tau \Gamma(\alpha)^{-1} \\
& =T \int_{-(t / T)}^{\infty}(t+T u)^{\alpha-1} g(u) d u \Gamma(\alpha)^{-1}
\end{aligned}
$$


Further, if $\alpha<\frac{1}{2}$

$$
\int\left|\tilde{I}_{\alpha} g_{T}(t)\right|^{2} d t=\frac{T^{1+2 \alpha}}{2 \pi} \int|\hat{g}(\lambda)|^{2}|\lambda|^{-2 \alpha} d \lambda
$$

where $\hat{g}$ is the Fourier transform of $\mathrm{g}$. Also

$$
\left|\tilde{I}_{\alpha} g_{T}(t)\right| \leqq K T^{\alpha}
$$

for all $t$ with $K$ an absolute constant.

The equality (12) follows straightforwardly on using the Parseval relation twice. One also notes that $\left(\tilde{I}_{\alpha} g_{T}\right)(t)$ is what one obtains on applying the operator $I_{a}$ to $g(-t / T)$. The inequality (15) is obtained by using the inequality

$$
\begin{aligned}
\left|\tilde{I}_{\alpha} g_{T}(t)\right| & \leqq \int_{-(t / T)}^{\infty} h(u) \frac{d u}{\Gamma(\alpha)} \\
& =\int_{(t / T)}^{(t / T)+1} h(u) \frac{d u}{\Gamma(\alpha)}+\int_{-(t / T)+1}^{\infty} h(u) \frac{d u}{\Gamma(\alpha)} \\
& \leqq K^{\prime} \int_{-(t / T)}^{-(t / T)+1} T(t+T u)^{\alpha-1} d u+T^{\alpha} \int|g(u)| d u \\
& \leqq K T^{\alpha} .
\end{aligned}
$$

A corresponding result can be given for fractional derivatives.

Lemma 3. Let $Y(t)$ be a separable weakly stationary process, $E Y(t) \equiv 0$, and $g(t)$ a bounded integrable function that satisfies a Lipschitz condition of fixed order $\beta, 0<\beta<1$, uniformly over $(-\infty, \infty)$. Then if $\left(D^{\alpha} Y\right)(t)$ exists, $0<\alpha<\beta$, and if $g_{T}(t)=g(t / T)$ it follows that

$$
\int\left(D^{\alpha} Y\right)(t) g_{T}(t) d t=\int Y(t)\left(\tilde{D}^{\alpha} g_{T}\right)(t) d t
$$

where

$$
\left(\tilde{D}^{\alpha} g_{T}\right)(t)=\int_{-t}^{\infty}(t+\tau)^{-\alpha} g\left(\frac{\tau}{T}\right) d \tau \Gamma(1-\alpha)^{-1}
$$

Moreover, if $\hat{g}(\lambda)|\lambda|^{\alpha} \in L^{2}$

$$
\int\left|\tilde{D}^{\alpha} g_{T}(t)\right|^{2} d t=\frac{T^{1-2 \alpha}}{2 \pi} \int|\hat{g}(\lambda)|^{2}|\lambda|^{2 \alpha} d \lambda
$$

Further,

$$
\left|\tilde{D}^{\alpha} g_{T}(t)\right| \leqq K T^{-\alpha}
$$

for all $t$ with $K$ an absolute constant. 
Relation (16) follows by the Parseval relation as in Lemma 2. Now

$$
\begin{aligned}
\Gamma(1-\alpha) \tilde{D}^{\alpha} g_{T}(t) & =\frac{d}{d t} \int_{-t}^{\infty}(t+\tau)^{-\alpha} g_{T}(\tau) d \tau \\
& =\frac{d}{d t} \int_{0}^{\infty} u^{-\alpha} \frac{d}{d u}\left\{G_{T}(u-t)-G_{T}(-t)\right\} d u
\end{aligned}
$$

where $G_{T}$ is the indefinite integral of $g_{T}$. On integrating by parts we obtain

$$
\begin{aligned}
\Gamma(1-\alpha) \tilde{D}^{\alpha} g_{T}(t) & =\frac{d}{d t} \int_{0}^{\infty} \alpha u^{-1-\alpha}\left\{G_{T}(u-t)-G_{T}(-t)\right\} d u \\
& =\int_{0}^{\infty} \alpha u^{-1-\alpha}\left\{g_{T}(u-t)-g_{T}(-t)\right\} d u .
\end{aligned}
$$

The expression

$$
\int_{0}^{\infty} u^{-1-\alpha}\left\{g_{T}(u-t)-g_{T}(-t)\right\} d u=T^{-\alpha} \int_{0}^{\infty} v^{-1-\alpha}\left\{g\left(v-\frac{t}{T}\right)-g\left(-\frac{t}{T}\right)\right\} d v
$$

Notice that the integral on the right-hand side of $(20)$ is bounded in absolute value by

$$
K \int_{0}^{1} v^{-1+\beta-\alpha} d v+\int_{-\infty}^{\infty}|g(v)| d v+\frac{1}{\alpha}\left|g\left(-\frac{t}{T}\right)\right| .
$$

The assumption that $g \in \operatorname{Lip}(\beta)$ has been used. $K$ is a constant.

The range within which the result of Lemma 3 holds can be broadened somewhat. Let us say that $g \in \Lambda_{\beta}, \beta>0$, if it is $[\beta]$ times continuously differentiable $([\beta]$ is the greatest integer less than or equal to $\beta)$ and its derivative $D^{[\beta]} g$ satisfies a Lipschitz condition of order $\beta-[\beta]$ uniformly over $(-\infty, \infty)$. The result of Lemma 3 then holds for $g$ a bounded integrable function in $\Lambda_{\beta}, \beta>0$, and $[\beta] \leqq \alpha<\beta$.

It is clear that results corresponding to Lemmas 2 and 3 for the more general context of fractional integrals (derivatives) centred at $\mu$ hold and can be derived by the same type of argument.

\section{A central limit theorem}

We can now obtain a central limit theorem for the sequence $A(T)$ as $T \rightarrow \infty$ under appropriate conditions by applying the main theorem of [5].

Theorem. Let $X(t)$ be a separable strictly stationary process that is the $\alpha$ th fractional integral for some $\alpha,|\alpha|<\frac{1}{2}$ of a process $Y(t)$ satisfying Assumption A. Then the spectral density $h(\lambda)$ of the process $X(t)$ is such that

$$
\lim _{\lambda \rightarrow 0} h(\lambda)|\lambda|^{2 \alpha}=f(0)>0
$$


where $f(0)$ is the value of the spectral density of the process $Y(t)$ at zero. Consider the sequence of random variables

$$
A(T)=\int X(t) g\left(\frac{t}{T}\right) d t
$$

as $T \rightarrow \infty$, where $g$ is a bounded integrable function. If $\alpha<0$ we also assume that $g$ satisfies a Lipschitz condition of order $\beta, 0<|\alpha|<\beta \leqq 1$, uniformly over $(-\infty, \infty)$. Then

$$
A(T) / \sigma(T)
$$

is asymptotically normally distributed with mean zero and variance $2 \pi f(0)$ as $T \rightarrow \infty$, where

$$
\sigma^{2}(T)=\frac{T^{1+2 \alpha}}{2 \pi} \int|\hat{g}(\lambda)|^{2}|\lambda|^{-2 \alpha} d \lambda .
$$

The remark on the spectral density $h(\lambda)$ of $X(t)$ near zero follows immediately from the fact that $X(t)$ is the $\alpha$ th fractional integral of the process $Y(t)$. By Lemmas 2 and $3 A(T)$ can be written in the form

$$
\int Y(t) \omega_{T}(t) d t
$$

with

$$
\omega_{T}(t)=\left(\tilde{I}_{\alpha} g_{T}\right)(t)
$$

if $0 \leqq \alpha<\frac{1}{2}$ and

$$
\omega_{\tau}(t)=\tilde{D}^{-\alpha} g_{T}(t)
$$

if $-\frac{1}{2}<\alpha \leqq 0$. Then

$$
\sigma^{2}(T)=W(T)=\int \omega_{T}(t)^{2} d t=\frac{T^{1+2 \alpha}}{2 \pi} \int|\hat{g}(\lambda)|^{2}|\lambda|^{-2 \alpha} d \lambda .
$$

Further,

$$
\lim _{T \rightarrow x} W(T)^{-1} \int \omega_{T}(t+h) \omega_{T}(t) d t=1
$$

for all $h$. The bounds obtained on $\tilde{I}_{\alpha} g_{T}(t), \tilde{D}^{-\alpha} g_{T}(t)$ in Lemmas 2 and 3 together with Assumption A imply that the conditions for the validity of the basic theorem of [5] are satisfied. Here $M(\lambda)$ is a jump function with total mass one at $\lambda=0$.

Notice that if $Y(t)$ satisfies Assumption A then the two derived processes (with $|\alpha|<\frac{1}{2}$ ) 


$$
\begin{aligned}
& \left(C_{\alpha, \mu} Y\right)(t)=\frac{1}{\Gamma(\alpha)} \int_{-\infty}^{t}(t-\tau)^{\alpha-1} \cos \mu \tau Y(\tau) d \tau \\
& \left(S_{\alpha, \mu} Y\right)(t)=\frac{1}{\Gamma(\alpha)} \int_{-\infty}^{t}(t-\tau)^{\alpha-1} \sin \mu \tau Y(\tau) d \tau
\end{aligned}
$$

are jointly stationary. Of course, these processes are just the real and imaginary parts of $\left(I_{\alpha, \mu} Y\right)(t)$. One can also remark that the processes $\left(C_{\alpha, \mu} Y\right)(t)$ and $\left(S_{\alpha, \mu} Y\right)(t)$ have spectral densities which behave like

$$
K|\lambda \pm \mu|^{-2 \alpha}
$$

in the neighborhood of $\pm \mu$ respectively with $K$ a constant. The following result can be regarded as a corollary of the theorem since it is obtained by essentially the same type of argument.

Corollary. Consider the processes

$$
\left(C_{\alpha_{j}, \mu_{j}} Y\right)(t), \quad\left(S_{\alpha_{j}, \mu_{j}} Y\right)(t)
$$

$\left|\alpha_{j}\right|<\frac{1}{2}, 0<\mu_{1}<\mu_{2}<\cdots<\mu_{m}, j=1, \cdots, m$ derived from a process $Y(t)$ satisfying Assumption A. Let $g_{i}(t), j=1, \cdots, m$, be bounded integrable functions. If $\alpha_{j}<0$ we also assume that $g_{i}(t)$ satisfies a Lipschitz condition of order $\beta_{j}$, $0<\left|\alpha_{i}\right|<\beta_{j} \leqq 1$. Let

$$
\begin{aligned}
& A_{j}(T)=\int\left(C_{\alpha_{i}, \mu_{j}} Y\right)(t) g_{j}\left(\frac{t}{T}\right) d t \\
& B_{i}(T)=\int\left(S_{\alpha_{i}, \mu_{j}} Y\right)(t) g_{j}\left(\frac{t}{T}\right) d t .
\end{aligned}
$$

Then

$$
A_{i}(T) / \sigma_{i}(T), \quad B_{i}(T) / \sigma_{i}(T)
$$

$j=1, \cdots, m$ are asymptotically normal and independent with mean zero and variance $\pi f\left(\mu_{i}\right)$, where

$$
\sigma_{i}^{2}(T)=\frac{T^{1+2 \alpha_{i}}}{2 \pi} \int\left|\hat{g}_{i}(\lambda)\right|^{2}|\lambda|^{-2 \alpha_{i}} d \lambda .
$$

In the context of the theorem, one could consider

$$
A(a T) / \sigma(T), \quad a>0,
$$

as a random process and it is clear that on heuristic grounds one would expect to have weak convergence as $T \rightarrow \infty$ to a Gaussian random process $Z(a)$ with mean zero and covariance function

$$
\operatorname{Cov}(Z(a), Z(b))=2 \pi f(0)\left\{|a|^{1+2 \alpha}+|b|^{1+2 \alpha}-|a-b|^{1+2 \alpha}\right\},
$$


$a, b>0$. This type of result has been obtained by Taqqu in [7] for partial sums of instantaneous functions of Gaussian stationary sequences under appropriate conditions. His result corresponds to $g(t)$ given by (2).

\section{Random solutions of Burgers equation}

In Section 6 of [6], a class of random solutions to the Burgers equation was considered. Some doubt was expressed as to the asymptotic normality of the solutions in the one-dimensional case. The question is related to some of the issues brought up in this paper. We shall remark again about the reasons for the doubt in [6], but shall show that under appropriate conditions one does in fact get asymptotic normality.

The Burgers equation for $u=u(t, x)$ is

$$
u_{t}+u u_{x}=\mu u_{x x}, \quad \mu>0 .
$$

Consider a random solution of (30), $u=2 \mu \Psi_{x}(c-\Psi)^{-1}$, with $c$ a positive constant and $\Psi(t, x)$ a solution of the heat equation $\phi_{t}=\mu \phi_{x x}$, strictly stationary in $x$ with

$$
\max _{x}\left|\Psi_{0}(x)\right|<c, \quad E \Psi_{0}(x) \equiv 0
$$

where $\Psi_{0}(x)=\Psi(x, 0)$. Let the spectral distribution function of $\Psi_{0}$ be $F(\lambda)$. Since $u_{0}(x)=u(0, x)=2 \mu \Psi_{0 x}\left(c-\Psi_{o}\right)^{-1}$, we require that $\int \lambda^{2} d F(\lambda)<\infty$. Notice that (31) implies that $\max _{x}|\Psi(x, t)|<c$ for all $t$. We also assume that $\Psi_{0}$ satisfies Assumption A and so is in particular strongly mixing. These conditions could be weakened somewhat but they are kept so as to be consistent with [6]. We are interested in the asymptotic distribution of $u(x, t)$ for fixed $x$ as $t \rightarrow \infty$ when it is appropriately centred and normalized. Since

$$
\Psi(x, t)=\int_{x}^{x}(4 \pi \mu t)^{-\frac{1}{2}} \exp \left\{-\frac{(x-y)^{2}}{4 \mu t}\right\} \Psi_{0}(y) d y
$$

it follows that $\max _{x}|\Psi(x, t)| \rightarrow 0$ as $t \rightarrow \infty$ and $u(x, t) \simeq 2 \mu c^{-1} \Psi_{x}$. But

$$
\Psi_{x}(x, t)=-\int(4 \pi \mu t)^{-\frac{1}{2}} \exp \left\{-\frac{(x-y)^{2}}{4 \mu t}\right\} \Psi_{0 y}(y) d y .
$$

Notice that $\Psi_{0 y}(y)$ is a strongly mixing process with spectral density $\lambda^{2} f(\lambda)$, $f(\lambda)=F^{\prime}(\lambda)$. In looking at (32) one is narrow band-pass filtering the process $\Psi_{0 y}(y)$ and this is a strongly mixing process whose spectral density has a high order zero at $\lambda=0$. Under such circumstances the usual form of central limit theorem need not hold, and this led to possible doubts about the asymptotic normality of (32). We give the following simple example. Consider a homogeneous process $\xi(t),-\infty<t<x$, with independent increments. In particular, one 
could take $\xi(t)$ to be a Poisson process. Set $X(t)=\xi(t)-2 \xi(t-1)+\xi(t-2)$. The process $X(t)$ is then a strongly mixing stationary process with covariance function

$$
R(t)=\left\{\begin{array}{lc}
2-3|t| & \text { if }|t| \leqq 1 \\
-2+|t| & \text { if } 1 \leqq|t| \leqq 2 \\
0 & \text { otherwise }
\end{array}\right.
$$

Notice that

$$
\int_{0}^{T} X(u) d u=\int_{T-1}^{T}\{\xi(u)-\xi(u-1)\} d u-\int_{0}^{1}\{\xi(u-1)-\xi(u-2)\} d u,
$$

so that the sequence (33) has a non-normal limiting distribution as $T \rightarrow \infty$ if one does not normalize at all.

Returning to (32), it is clear that one can write it as

$$
\Psi_{x}(x, t)=\int \Psi_{0}(y)(4 \pi \mu t)^{-\frac{1}{2}} \frac{\partial}{\partial y} \exp \left\{-\frac{(x-y)^{2}}{4 \mu t}\right\} d y .
$$

Without loss of generality set $x=0$. In this form one can see that the theorem of [5] is applicable and so one does have asymptotic normality. The key is that we are dealing with an expression of the form

$$
\int g\left(\frac{t}{T}\right) \frac{d}{d t} X(t) d t
$$

with $X(t)$ a process satisfying Assumption A. If $g$ is absolutely continuous with $\lim _{|t| \rightarrow \infty} g^{\prime}(t)=0$ and $g, g^{\prime}$ absolutely integrable, (34) can be rewritten as $-(1 / T) \int g^{\prime}(t / T) X(t) d t$ and the expression is asymptotically normal as $T \rightarrow \infty$. In the case of (33), the function $g$ is not differentiable.

The global asymptotic behavior of the whole process (32) can be determined as $T \rightarrow \infty$. However, that would be better pursued elsewhere.

\section{References}

[1] Davydov, Yu. A. (1970) The invariance principle for stationary processes. Theor. Prob. Appl. 15, 487-498.

[2] Helson, H. and Sarason, D. (1967) Past and future. Math. Scand. 21, 5-16.

[3] MANDELBROT, B. B. (1975) Limit theorems on the self-normalized range for weakly and strongly dependent processes. $Z$. Wahrscheinlichkeitsth. 31, 271-285.

[4] MCLEISH, D. L. (1975) Invariance principles for dependent variables. Z. Wahrscheinlichkeitsth. 32, 165-178.

[5] Rosenblatt, M. (1961) Some comments on narrow band-pass filters. Quart. Appl. Math.18, 387-393.

[6] Rosenblatt, M. (1968) Remarks on the Burgers equation. J. Math. Phys. 9, 1129-1136.

[7] TAQQU, M. (1975) Weak convergence to fractional Brownian motion and to the Rosenblatt process. Z. Wahrscheinlichkeitsth. 31, 287-302.

[8] Zygmund, A. (1968) Trigonometric Series. Cambridge University Press. 